\title{
ACUMULAÇÃO DE MATÉRIA SECA DO TOMATEIRO CULTIVADO EM SUBSTRATO COM DIFERENTES DOSES DE FERTILIZANTES ${ }^{1}$
}

\author{
DRY MATTER ACCUMULATION OF TOMATO PLANTS GROWN IN SUBSTRATE \\ UNDER DIFFERENT FERTILIZER DOSES
}

\author{
Roberto André Grave ${ }^{2}$ Jerônimo Luiz Andriolo ${ }^{3}$ Hardi René Bartz ${ }^{4}$
}

\section{- NOTA -}

RESUMO

\footnotetext{
Plantas de tomateiro foram cultivadas em substrato no interior de um ambiente protegido localizado no Departamento de Fitotecnia da UFSM, em Santa Maria, RS, com o objetivo de determinar o efeito de cinco doses de fertilizantes sobre a acumulação da matéria seca da parte vegetativa e dos frutos. Foram efetuados três experimentos, no outono-inverno de 1997, na primavera-verão de 1997 e no outono-inverno de 1998, empregando os híbridos Diva, Monte Carlo e Saladette, respectivamente. Foi utilizada como referência uma dose semanal com as seguintes quantidades de fertilizantes, em $\mathrm{g}$ por planta: 4 de $\mathrm{KNO}_{3} ; 6,3$ de $\mathrm{Ca}\left(\mathrm{NO}_{3}\right)_{2} ; 1,5$ de superfosfato simples; 3 de $\mathrm{MgSO}_{4}$, complementada por 0,13m $\ell$ de ferro quelatizado $(5 \%$ de $\mathrm{Fe}$ ) e 0,7mL de uma solução contendo os demais micronutrientes. No experimento 1 , a dose de $T 1$ foi igual àquela de referência e as doses de T2 e T3 foram obtidas multiplicando-se a dose de referência por 1,15 e 1,3, respectivamente. Nos experimentos $2 e$ 3 , as doses de T1, T2 e T3 corresponderam à dose de referência multiplicada por 0,6; 1,15 e 1,7, respectivamente. Para cada um dos dois híbridos e nos três experimentos, as plantas foram localizadas em três blocos, com três fileiras de 35 plantas em cada bloco. Cada tratamento foi aplicado uma vez por semana a todas as plantas da mesma fileira, através da fertirrigação. Foi determinada a matéria seca acumulada na parte vegetativa da planta e nos frutos no início do período de maturação dos mesmos, em cada experimento. Não foram observadas diferenças significativas na acumulação de matéria seca na parte vegetativa da planta e nos frutos entre as três doses e nos três experimentos.
}

Palavras-chave: Lycopersicon esculentum, rendimento, nutrientes, fertirrigação.

SUMMARY

Tomato plants were grown in substrate in a greenhouse at Departamento de Fitotecnia, Universidade Federal de Santa Maria, RS, Brazil, to determine the effect of five fertilizer levels on dry matter accumulation of vegetative plant parts and fruits. Three experiments were done, in autumnwinter/1997, spring-summer/1997 and autumn-winter/1998, using hybrids Diva, Monte Carlo and Saladette, respectively. The following quantities of fertilizers were used as control, in g/plant, supplied once a week to each plant: $\mathrm{KNO}_{3}, 4.0 ; \mathrm{Ca}\left(\mathrm{NO}_{3}\right)_{2}, 6.3$; superphosphate $\left(20 \% \mathrm{P}_{2} \mathrm{O}_{5}\right), 1.5 ; \mathrm{MgSO}_{4}, 3.0$. Micronutrients were added by $0.13 \mathrm{~m} \ell$ of an iron chelate $(5 \% \mathrm{Fe})$ and $0,7 \mathrm{~m} \ell$ of a commercial mixture. In experiment 1, quantities of fertilizers of $T 1$ were equal to that of the reference, but $T 2$ and $T 3$ were established by multiplying the quantities from $T 1$ by 1.15 and 1.3, respectively. In experiments 2 and 3, quantities of $T 1$ were multiplied by $0.6 ; 1.15$ and 1.7 in treatments $T 1, T 2$ and T3, respectively. For experimental design, plants of each hybrid were plotted in three blocks, with three rows and 35 plants each block, all plants of a row receiving once a week the same quantities of fertilizers by fertigation. The dry matter accumulation of vegetative plant parts and fruits was determined at the stage when the first fruits begun to ripe. Significant differences in dry matter accumulation of both vegetative parts and fruits among treatments were not observed, in all experiments.

\footnotetext{
${ }^{1}$ Parte da dissertação de mestrado apresentada pelo primeiro autor ao Programa de Pós-graduação em Agronomia, Centro de Ciências Rurais, Universidade Federal de Santa Maria (UFSM), RS.

${ }^{2}$ Engenheiro Agrônomo, Mestre em Agronomia.

${ }^{3}$ Engenheiro Agrônomo, Doutor, Professor Titular, Departamento de Fitotecnia, UFSM, 97105-900, Santa Maria, RS. Bolsista CNPq. E-mail: andriolo@creta.ccr.ufsm.br. Autor para correspondência.

${ }^{4}$ Engenheiro Agrônomo, Mestre, Professor Assistente, Departamento de Solos, CCR, UFSM.
} Recebido para publicação em 18.01.00. Aprovado em 03.01.00 
Key words: Lycopersicon esculentum, yield, nutrients, fertigation.

O cultivo do tomateiro em substrato é uma das principais alternativas de escape tanto para as moléstias do sistema radicular, como para os problemas decorrentes da concentração excessiva de nutrientes na camada superficial do solo, que conduzem à salinização (INRA, 1983; BLANC, 1987; ANDRIOLO, 1999). O procedimento de rotina empregado nos países do Hemisfério Norte que já utilizam essa técnica há muitos anos não faz qualquer distinção entre as necessidades hídricas e minerais das plantas (JEANNEQUIN, 1987. O uso desta técnica no Brasil encontra limitações de ordem econômica, devido ao alto custo dos fertilizantes solúveis. Para reduzir esses custos, ANDRIOLO \& POERSCHKE (1997) desenvolveram um método para o cultivo do tomateiro em substrato, empregando materiais orgânicos e suprimento de nutrientes a intervalos semanais, através de uma solução nutritiva completa. Os resultados obtidos quanto ao rendimento de frutos foram semelhantes àqueles observados no cultivo tradicional no solo (ANDRIOLO et al. 1997. Entretanto, ainda não foram encontrados na literatura nacional resultados de pesquisas sobre o comportamento do tomateiro cultivado em substrato no qual se empregaram doses de nutrientes diferentes das recomendadas por aqueles autores. $\mathrm{O}$ objetivo deste trabalho foi determinar o efeito de diferentes doses de $\mathrm{N}$ e $\mathrm{K}$ sobre o crescimento dos órgãos vegetativos aéreos e dos frutos do tomateiro cultivado em substrato, empregando a fertirrigação semanal, segundo o procedimento de rotina recomendado aos produtores.

Três experimentos foram realizados no interior de uma estufa de polietileno de $400 \mathrm{~m}^{2}$, localizada na área experimental do Departamento de Fitotecnia da UFSM, RS, no outono-inverno (experimento 1) e primavera-verão (experimento 2) do ano de 1997 e no outono-inverno (experimento 3) do ano de 1998. No experimento 1, foi empregado o híbrido indeterminado Diva, com semeadura em 20/02/1997 e término em 04/07/1997. No segundo experimento, foi usado o híbrido indeterminado Monte Carlo, com semeadura em 22/08/1997 e término em 19/12/1997. No terceiro experimento, utilizou-se o híbrido semideterminado Saladette, com semeadura em 09/02/98 e término em 20/06/98.

As mudas foram transplantadas para $o$ interior de sacolas plásticas, contendo substrato comercial (Plantmax folhosas), na densidade de 2,8 plantas $\mathrm{m}^{-2}$, em fileiras simples $(1,2 \mathrm{~m}$ entre fileiras e $0,30 \mathrm{~m}$ entre plantas). O volume total de água contida no substrato foi de $2,5 \ell$, retido em um volume de material seco igual a $3,7 \ell$. Foi utilizada como referência a dose semanal recomendada por ANDRIOLO \& POERSCHKE (1997) para o cultivo do tomateiro em substrato, com as seguintes quantidades de fertilizantes, em g por planta: 4 de $\mathrm{KNO}_{3} ; 6,3$ de $\mathrm{Ca}\left(\mathrm{NO}_{3}\right)_{2} ; 3$ de $\mathrm{MgSO}_{4} ; 1,5$ de super fosfato simples, complementada por $0,13 \mathrm{~m} \ell$ de ferro quelatizado $(5 \%$ de $\mathrm{Fe})$ e $0,7 \mathrm{~m} \ell$ de uma solução contendo os demais micronutrientes. No experimento 1 , a dose de $\mathrm{T} 1$ foi igual àquela de referência e aquelas de T2 e T3 foram obtidas multiplicando-se a dose de referência por 1,15 e 1,3, respectivamente. Nos experimentos 2 e 3, as doses de T1, T2 e T3 corresponderam à dose de referência multiplicada por 0,$6 ; 1,15$ e 1,7 , respectivamente. Para cada um dos dois híbridos e nos três experimentos, as plantas foram localizadas em três blocos, com três fileiras de 35 plantas em cada bloco, e cada tratamento foi aplicado uma vez por semana a todas as plantas da mesma fileira, através da fertirrigação. No início do período de maturação dos frutos, foi determinada a acumulação de matéria seca da parte vegetativa da planta e dos frutos em cada experimento.

Não foram observadas diferenças significativas na acumulação de matéria seca da parte vegetativa da planta e dos frutos entre as três doses e nos três experimentos (Tabela 1). A fração média da matéria seca total alocada para os frutos foi de $30 \%$. Os resultados indicaram que o aumento das doses de fertilizantes na formulação atualmente

Tabela 1 - Produção de matéria seca (MS, g/pl.) da parte vegetativa da planta e de frutos de tomateiro cultivado em substrato com diferentes doses semanais de fertilizantes, em cada um dos experimentos realizados. UFSM, Santa Maria, 1997/98* .

\begin{tabular}{lrcc}
\hline Tratamentos & Experimento 1 & Experimento 2 & Experimento 3 \\
\hline Parte vegetativa & & & \\
T 1 & $262,34 \mathrm{a}$ & $271,23 \mathrm{a}$ & $232,56 \mathrm{a}$ \\
T 2 & $269,58 \mathrm{a}$ & $302,50 \mathrm{a}$ & $256,05 \mathrm{a}$ \\
T 3 & $260,66 \mathrm{a}$ & $332,05 \mathrm{a}$ & $289,80 \mathrm{a}$ \\
Frutos & & & \\
T 1 & $126,60 \mathrm{a}$ & $116,67 \mathrm{a}$ & $106,93 \mathrm{a}$ \\
T 2 & $118,76 \mathrm{a}$ & $122,33 \mathrm{a}$ & $105,90 \mathrm{a}$ \\
T 3 & $118,27 \mathrm{a}$ & $126,20 \mathrm{a}$ & $118,90 \mathrm{a}$ \\
\hline
\end{tabular}

* Médias seguidas pela mesma letra não diferem entre si pelo teste de Duncan, em nível de $5 \%$ de probabilidade de erro. 
empregada na fertirrigacão do tomateiro cultivado em substrato não aumenta a produção de frutos. Entretanto, para determinar a curva de resposta dessa cultura à adubação, faz-se necessário conduzir novos experimentos, empregando incrementos maiores entre os tratamentos, acima e abaixo da dose de referência atualmente recomendada.

\section{REFERÊNCIAS BIBLIOGRÁFICAS}

ANDRIOLO, J.L., POERSCHKE, P.L. Cultivo do tomateiro em substratos. Santa Maria : UFSM, Centro de Ciências Rurais, 1997. 12p. (Informe Técnico, 2).
ANDRIOLO, J.L. Fisiologia das culturas protegidas. Santa Maria : UFSM, 1999. 142p.

ANDRIOLO, J.L., DUARTE, T.S., LUDKE, L. $\boldsymbol{e} \boldsymbol{t} \boldsymbol{a l}$. Crescimento e desenvolvimento do tomateiro cultivado em substrato com fertirrigação. Horticultura Brasileira, v.15, n.1, p.28-32, 1997.

BLANC, D. Nutrition hyrique et minérale. In: BLANC, D. Les cultures hors sol. Paris : INRA, 1987. p.109-121.

INRA. Institut National de la Recherche Agronomique. L'INRA et les cultures sous serre. Paris : INRA, 1983. 230p.

JEANNEQUIN, B. Conduite de la fertilisation des cultures hors sol en maraîchage. PHM Revue Horticole, v.275, p.19-28, 1987.

Ciência Rural, v. 31, n. 5, 2001. 